\title{
Research on the Influencing Factors of SMEs Cluster Network Structure Evolution
}

\author{
Shujiang Yu \\ School of Economics and Management, \\ Hebei University of Technology, \\ Tianjin, China \\ e-mail:mbaysj@163.com
}

\author{
Guiyun Ding \\ School of Economics and Management, \\ Hebei University of Technology, \\ Tianjin, China \\ e-mail: dinggy124311@yahoo.com.cn
}

\begin{abstract}
This dissertation analyzes the effect of government ability, market demand, knowledge spillover, collective learning, influences of the leading enterprises, technical complexity and enterprise heterogeneity on the SMEs cluster network structure evolution according to the development characteristics of SMEs cluster network structure. We put forward relative research hypothesis, and then take three stages of development of wood-based panel enterprises cluster network structure in Wen'an for instance to verify the research hypothesis.
\end{abstract}

Keywords-enterprise cluster; network structure; characteristics of evolution; motivations of evolution

\section{INTRODUCTION}

In the past two years' researches, network analysis method is increasingly used in enterprises cluster. Network Analysis is an analytical tool which derived from the social network analysis, and emphasizes the relationship between the various nodes that are composed of the network analysis, and this method adapt to the complexity characteristic of the economic environment which today's enterprises cluster are in. However, in view of the existing literature, most domestic researches devote to the network structure and risk studies [1,2], the relationship between network structure and competitiveness of enterprises [3], the cluster network structure characteristics and functional analysis $[4,5,6]$. Few people research on cluster network structure evolution. If we can use a unified theoretical framework and analyze the motivation of evolution and influence factors of the SME cluster network structure based on the dynamic perspective of the evolution of enterprises cluster, it will further enrich the research in this field.

\section{REVIEW}

\section{A. Network Structure Evolution}

Wei Jiang[7] and many other people divide network structure of the enterprises cluster into three levels, namely as core network, auxiliary network, and perimeter network. Chen Huan[8] elaborated the network structure evolution of the enterprise cluster by different angles and cases. In germination stage: Cluster nodes are incomplete; the relationship between network nodes is weak; there is a one-to-one strong relationship between certain businesses nodes in the cluster. In growth stage: cluster area expanded, and the enterprise node and connection number increased gradually; the weak ties with the outside world cluster business or organization become strong gradually, and some large enterprises will eventually establish strong ties with the outside world; enterprises within the cluster build weak ties between. In maturity stage: node elements in network have been very complete; there are a lot of nodes, and the increase in the number has been gradually reduced; cooperate with big company, improve the level of technology, integrated into a larger market, and also has a wide range of weak link with the outside world; form a number of core enterprise (multi-axis), and the links between enterprises become weak; Social networks and local production networks expand outward, enterprises cluster has been developed to a considerable scale of production, competitive differentiation which key point is innovation become the focus of market competition rather than the most basic price competition, and the whole cluster of network transfers to innovation network. In degenerating stage, once the cluster has signs of recession or began to decline, if there is no timely adjust strategy, cluster competitiveness and cohesion will decline rapidly. The nodes in the cluster number is no longer increasing, even declined dramatically, and contact between enterprises becomes fierce lead to no longer close due to the competition.

\section{B. The Cluster Network Dimensions}

A large number of scholars has studied the network dimension, but there is no a unified theory. NAHAPIET $\mathbf{J}$ and GHOSHAL $S$ maintain that network structure includes attributes of the network connection, network density, network connectivity, network hierarchy. SIU and the supporters insist that network structure dimension includes network size and network center. Ren Sheng-gang and the supporters [9] insist network structure dimension includes networking body, networking strength and network structure properties, and network structure properties includes network size, network density, the stability of the network and node centrality. Dong Bao-bao [10] insists that network structure dimension includes network strength, network density and network centrality. Xie Zhen-dong [11] maintains corporate social network mainly includes four aspects of network centricity, network dynamics, networking strength and network heterogeneity. Wang Yue-hua [12] insists network structure includes three dimensions of network centricity, networking strength and network heterogeneity. In this paper, we choose three dimensions of network centricity, networking strength and network heterogeneity to measure the network structure of the cluster. 


\section{Network Evolution Dynamics}

Domestic scholars have carried on the preliminary exploration about evolution dynamics of the cluster network structure. Different network element such as knowledge spillovers, network culture, entrepreneurship, learning mechanisms plays a different role in each stage of the cluster evolution. But in each stage, the presence of weavers has played an important role. And its degree of organization of the internal network also seriously affected the ability of the enterprise cluster network upgrade. Tian Gang and the supporters discussed the evolution of innovation network, and maintain there are the influence factors of the cluster innovation network evolution: innovation subject knowledge difference in size, the degree of technical specialization and implicit or explicit knowledge transfer mode. Zhu Hai-yan and Wei Jiang based on the perspective of knowledge intensive services embedded analyzed that the cluster network evolution dynamic factors can be subdivided into four aspects of the heterogeneity of the bridge construction, "weak" power of the joint, the formation and evolution of the multi-level service system, and "cognitive balance" psychological .

\section{RESEARCH HYPOTHESIS}

\section{A. External Motivations of Cluster Network Structure Evolution}

$\mathrm{H} 1$ : There is a correlation between government ability and changes in enterprise cluster network structure.

$\mathrm{H} 2$ : There is a correlation between market demand and changes in enterprise cluster network structure.

\section{B. Internal Motivations of Cluster Network Structure Evolution}

H3: There is a correlation between knowledge spillovers and changes in enterprise cluster network structure.

H4: There is a correlation between collective learning and changes in enterprises cluster network structure.

H5: There is a correlation between the influences of leading enterprises and changes in enterprise cluster network structure.

H6: There is a correlation between technical complexity and changes in enterprise cluster network structure.

H7: There is a correlation between enterprise heterogeneity and changes in enterprise cluster network structure.

\section{SAMPLE SELECTION}

This empirical research object chooses Wen'an wood-based panel enterprises cluster and the questionnaires samples are primarily Zuo'gezhuang wood-based panel enterprises.At the same time, we also interviewed with Wen' an's development and reform commission, Zuo'gezhuang wood-based panel association in order to understand the overall situation of clusters better. To ensure that the samples are much more persuasive, most enterprises we chose established early with good momentum of development. They are mainly located in Zuo'gezhuang, and there are seven or eight leading enterprises in the cluster. We selected two leading enterprises and started to seek relationship between enterprises by leading enterprises from top and bottom. Determined 60 samples, these enterprises' information can represent the actual development situation of the cluster. The changes of their choices for partners can reflect the changes of the cluster network structure. In addition, in order to increase the reliability of the questionnaires, people filling in the questionnaires are basically in high-level leadership to guarantee clear understanding for enterprise's strategy, location in the cluster location, and so on.

The issuance of the questionnaires was done with the help of Wen'an's development and reform commission. We visited multiple enterprises and questionnaires were filled in time and back in time. We handed out 60 questionnaires and collected 60 . We got 54 effective questionnaires getting rid of invalid ones. Because some enterprises were established after 1992, not all questionnaires can include germination stage, growth stageand mature stage across 20 years of three stages of development and changes. According to statistics, there are 54 samples in 2012, 49 in 2002 and 47 in1992.

For space reasons, we mainly choose related data of enterprise cluster in maturity stage for empirical analysis.

\section{MAIN CONCLUSIONS}

Influencing factors of network center are government ability, knowledge spillover and collective learning.In past decade, government organization established industry association and issued a series of policies on relevant taxes and environmental protection. The establishment of industry association makes enterprises more familiar with each other. More often, government policies make large and medium-sized enterprises getting more benefits, and more enterprises are willing to get contacts with government in order to get their own place. Therefore, government ability and node centricity are positively correlated in this stage. Knowledge spillover and collective learning occur in all stages of cluster development, and thus influence the network node centricity positively as ever.

The influencing factor of networking strength is leading enterprises. With years of development, the number of leading enterprises in the cluster increased, and leading enterprises need more supporting enterprises. Leading enterprises are demanding more, so SMEs need to strengthen the communication with them to ensure that they are both satisfied with the products.

The influencing factor of network heterogeneity is knowledge spillovers and enterprises heterogeneity. Not only the explicit knowledge spillover, but also the tacit knowledge spillover works in this period. Knowledge reflected in daily work and study life allow enterprises to understand their own gap, and at this point associations and research institutions have set up in cluster, thus a large number of enterprises within the cluster started to cooperate and learn with these innovative organizations within the cluster and even the university and advanced enterprise outside the cluster, showing the enterprise cluster network heterogeneity. As the market segmentation and differences of products, technology is also getting more complicated, and enterprises have showed the heterogeneity. The emergence of heterogeneity required the enterprises to 
find new suppliers and customers, and even some organizations outside the cluster to establish contact with them. So enterprise heterogeneity is positive correlation.

In addition, the relationship between market demand, technical complexity and cluster network structure has not been verified. Market demand for non-significant has little impact on the evolution of the network structure, and that is probably because the market has already saturated in this period. That also may be because the sample size is too small to detect the relationship between them. We believe that the technology complexity should play a role in the evolution of the cluster network structure in maturity stage, and the non-significant results may be because the technology complexity has affected the other dimensions of network structure, also may be because the technologies of selected sample companies have been mature in mature stage, and the influences on the network structure evolution is too little to detect.

To sum up, in mature stage of cluster development, it is knowledge spillover that plays a most important role in evolution of Wen'an wood-based panel enterprises cluster network structure, followed by leading enterprises and the influence of government ability, and then the collective learning and enterprise heterogeneity.

\section{REFERENCES}

[1] Wang Fa-ming, Cai Ning and Zhu Hao-yi, " The research on Cluster network structure and risk " China University of Geosciences, 2006(06)

[2] Cai Ning, Wu Jie-bing and Yin Ming, " Anlaysis of structure and function of the complex networks clusters structure " Economic Geography, 2006, (5)

[3] Xie Hong-ming and Liu Shao-chuan, " The research on the Relationship between Industrial clusters, networking and enterprise competitiveness ", Engineering and Engineering Management, 2007, (2)

[4] Ye Li-juan Kuang Guo-liang, "Analysis of the characteristics of enterprises network in cluster ", Reformation \& Strategy, 2009, (11)

[5] Kuang Guo-liang and Yang Shui-chang, " Analysis of corporate network of industrial clusters in the mode of government-led ", Reformation \& Strategy, 2008, (11)

[6] Liu Bian-sheng and Wang Kai, " Studies on enterprise cluster network structure and performance " , Industrial Technology \& Economy, 2007, (09)

[7] Wei Jiang, "Innovative systems and technology learning ", Beijing: Science Press, 2003

[8] Chen Huan and Wang Se, "Researches on industrial cluster life cycle based on the corporate network structure ", Technology and market, 2008 (08)

[9] Ren Sheng-li and Li Li, " Enterprise network structure dimension and its impact on innovation performance " , Management Modernization in China,2208-2218

[10] Dong Bao-bao," Relationship between network structure and competitive advantage based on the mediating effect of dynamic capabilities", Journal of Management, 2012 (01)

[11] Xie Zhen-dong, "Relationship between industrial clusters enterprises social network and entrepreneurial performance in industrial clusters ", Zhejiang University, 2007

[12] Wang Yue-hua, " Relationship between corporate social network and the growth of SMEs " , Zhejiang University of Technology, 2009 